\author{
RESEARCH ARTICLE \\ 10.1029/2018JC014586 \\ Key Points: \\ - Tidal harmonics comprise about half \\ of the power in the deep ocean \\ pressure field over $400-1,000 \mu \mathrm{Hz}$ \\ - Solar pressure or p-modes are \\ readily identified throughout the \\ $400-$ to $4,000-\mu \mathrm{Hz}$ bands, both by \\ their characteristic frequencies and \\ through their pervasive \\ nonstationarity \\ - Two previously unrecognized \\ sources of infragravity wave \\ variability have been characterized, \\ indicating the complexity of the \\ arguably least studied class of waves \\ in the deep ocean
}

Supporting Information:

- Supporting Information S1

Correspondence to:

A. D. Chave,

achave@whoi.edu

Citation:

Chave, A. D., Luther, D. S., \& Thomson, D. J. (2019). High-Q spectral peaks and nonstationarity in the deep ocean infragravity wave band: Tidal harmonics and solar normal modes. Journal of Geophysical Research: Oceans, 124, 2072-2087. https://doi. org/10.1029/2018JC014586

Received 18 SEP 2018 Accepted 18 FEB 2019 Accepted article online 20 FEB 2019 Published online 28 MAR 2019

(c)2019. American Geophysical Union. All Rights Reserved.

\section{High- $Q$ Spectral Peaks and Nonstationarity in the Deep Ocean Infragravity Wave Band: Tidal Harmonics and Solar Normal Modes}

\author{
Alan D. Chave ${ }^{1}$ (D), Douglas S. Luther ${ }^{2}$ (D) and David J. Thomson ${ }^{3}$ \\ ${ }^{1}$ Department of Applied Ocean Physics and Engineering, Woods Hole Oceanographic Institution, Woods Hole, MA, USA, \\ ${ }^{2}$ Department of Oceanography, JIMAR and SOEST, University of Hawai'i at Mānoa, Honolulu, HI, USA, ${ }^{3}$ Department of \\ Mathematics and Statistics, Queen's University, Kingston, Ontario, Canada
}

Abstract Infragravity waves have received the least study of any class of waves in the deep ocean. This paper analyzes a 389-day-long deep ocean pressure record from the Hawaii Ocean Mixing Experiment for the presence of narrowband $(\lesssim 2 \mu \mathrm{Hz}$ ) components and nonstationarity over $400-4,000 \mu \mathrm{Hz}$ using a combination of fitting a mixture noncentral/central $\chi^{2}$ model to spectral estimates, high-resolution multitaper spectral estimation, and computation of the offset coherence between distinct frequencies for a given data segment. In the frequency band $400-1,000 \mu \mathrm{Hz}$ there is a noncentral fraction of $0.67 \pm 0.07$ that decreases with increasing frequency. Evidence is presented for the presence of tidal harmonics in the data over the $400-$ to $1,400-\mu \mathrm{Hz}$ bands. Above $\sim 2,000 \mu \mathrm{Hz}$ the noncentral fraction rises with frequency, comprising about one third of the spectral estimates over 3,000-4,000 $\mu \mathrm{Hz}$. The power spectrum exhibits frequent narrowband peaks at 6-11 standard deviations above the noise level. The widths of the peaks correspond to a $Q$ of at least 1,000, vastly exceeding that of any oceanic or atmospheric process. The offset coherence shows that the spectral peaks have substantial ( $p=0.99-0.9999)$ interfrequency correlation, both locally and between distinct peaks within a given analysis band. Many of the peak frequencies correspond to the known values for solar pressure modes that have previously been observed in solar wind and terrestrial data, while others are the result of nonstationarity that distributes power across frequency. Overall, this paper documents the existence of two previously unrecognized sources of infragravity wave variability in the deep ocean.

Plain Language Summary Infragravity waves (IGWs) have arguably received the least study of any class of waves in the deep ocean. This paper contains the analysis of a 389 day long deep ocean pressure record from northwest of Hawaii for the presence of narrowband $(\lesssim 2 \mu \mathrm{Hz})$ components and nonstationarity over $400-4000 \mu \mathrm{Hz}$. Evidence is presented for the presence of tidal harmonics in the data over the 400-1400 $\mu \mathrm{Hz}$ band, possibly originating in the Gulf of Alaska. The power spectral density exhibits frequent narrowband peaks at 6-11 standard deviations above the noise level. Some of the peak frequencies correspond to the known values for solar normal modes that have previously been observed in solar wind, seismic, geomagnetic, barometric and ionospheric data, while others are the result of nonstationarity that distributes power across frequency. Overall, this paper documents the existence of two heretofore unrecognized sources of IGW variability in the deep ocean.

\section{Introduction}

Deep ocean infragravity waves (IGWs) are surface gravity waves that occur at frequencies higher than rotation-dependent, tidal gravity waves $(\gtrsim 100 \mu \mathrm{Hz})$ and at frequencies smaller than those of wind-generated swell and sea waves $(\lesssim 40,000 \mu \mathrm{Hz})$. Commonly, the term IGW is used for gravity waves at frequencies of $40,000 \mu \mathrm{Hz}$ down to $8,000 \mu \mathrm{Hz}$ or even $3,000 \mu \mathrm{Hz}$, depending on the author. By analogy with the electromagnetic spectrum, the term far-IGW has been used to refer to IGWs at frequencies smaller than $3,000 \mu \mathrm{Hz}$ down to as low as $\sim 100 \mu \mathrm{Hz}$. In this paper, the term IGW will be used to refer to the entire band of frequencies from 100 to $40,000 \mu \mathrm{Hz}$.

Like swell waves, pelagic IGWs have a depth-dependent character in the deep ocean (defined as $\sim 5-\mathrm{km}$ depth) until their frequency falls below about $3,000 \mu \mathrm{Hz}$, at which point their horizontal velocity and pressure fields are essentially depth-independent. Pelagic IGWs generally propagate horizontally as isotropic free 
waves, dispersing like linear gravity waves, but near-coast bathymetry can refract and trap them, forming edge waves (e.g., Munk et al., 1964). Pelagic IGWs, including tsunamis, occur at basin scales with very little attenuation due to their large wavelengths (one to hundreds of kilometers) and small water velocities. Because of their scale, they provide a mechanism to couple processes between the ocean, solid earth, and atmosphere.

While IGWs have been extensively studied in the nearshore zone (e.g., Elgar et al., 1992), they have arguably received the least study of any class of waves in the deep ocean, particularly at the frequencies (400$4,000 \mu \mathrm{Hz}$ ) in this study. Webb et al. (1991) provide observational evidence that deep water IGWs are predominantly free waves radiating from nearshore regions where energy is transferred nonlinearly from the higher frequency swell waves to IGWs, although their work is focused on frequencies at the upper end (and beyond) of the frequency band studied here. With a numerical model, Uchiyama and McWilliams (2008) showed that while coastal sources of IGWs are strong, it is still plausible that some of the open ocean IGW energy exists as forced waves from locally interacting swell. All of the IGWs that are forced by swell in some manner can be expected to have a seasonal dependence, in tune with the swell wave energy in each hemisphere, as has been demonstrated by Aucan and Ardhuin (2013). Godin et al. (2013) proposed a universal spectrum model for these higher frequency IGWs, with which the pressure spectra presented here are in approximate agreement.

At lower frequencies of $\sim 100-1,000 \mu \mathrm{Hz}$, seasonal variability of pelagic IGWs is very small (e.g., Aucan \& Ardhuin, 2013; Filloux et al., 1991), suggesting the dominance of an energy source unrelated to atmospheric forcing. Luther et al. (2014) postulated that a nonlinear cascade of tidal energy into the low-frequency end of the IGW band in the coastal zone results in IGW leakage out into the pelagic oceans. Propagation of the M4 overtide from the Argentine Basin into the Atlantic Ocean has been documented by Ray (2007), so there is no question that back flux into the open ocean occurs from shallow regions.

IGWs have received recent attention due to the discovery of a persistent low-frequency hum in seismic records (Nawa et al., 1998), putatively caused by persistent excitation of fundamental mode spheroidal oscillations of Earth. These have been variously attributed to resonant oscillations between the solid earth and atmosphere (Nishida et al., 2000), atmosphere/ocean/seafloor coupling by IGWs (e.g., Rhie \& Romanowicz, 2004), and interacting IGWs across the continental shelves or in the pelagic ocean (Webb, $2007,2008)$. The analyses in all of these studies utilize record lengths of $\mathrm{O}(1$ day), yielding a resolution bandwidth of $\mathrm{O}(20 \mu \mathrm{Hz})$, presuming that a reliable data taper is employed. As will be shown in this paper, the deep ocean pressure spectrum in a significant fraction of the IGW band is populated by high-Q peaks with a bandwidth of $2 \mu \mathrm{Hz}$ or less that cannot be resolved using 1-day data sections. It is not the purpose of this paper to investigate the earth hum problem in detail, but it must be noted that no evidence for the presence of terrestrial spheroidal normal modes was found in any of the analyses carried out in the course of this work.

Thomson et al. (2007) and Thomson and Vernon $(2015,2016)$ presented a comprehensive analysis of a wide range of terrestrial data, including seismic, geomagnetic, barometric, and interplanetary ones, which showed that their spectra are dominated by narrowband, high- $Q$ modes whose frequencies and behaviors are consistent with normal modes of Sun at frequencies above $\sim 250 \mu \mathrm{Hz}$. Such modes on Earth will be of planetary scale given their origin. Those works build on Thomson et al. (1995, 2001), where it was demonstrated that solar normal modes are present in the solar wind and interplanetary magnetic field propagating out from Sun.

It is the purpose of this paper to investigate the spectral characteristics of a deep ocean pressure record over the frequency band $400-4,000 \mu \mathrm{Hz}$, including evaluation of the null hypotheses that the observed characteristics are inconsistent with forcing by solar modes (tidal harmonics) against the alternate hypothesis that they are due to the presence of normal modes (tidal harmonics). Section 2 summarizes the data analysis methods that will be utilized. Section 3 presents the data, and section 4.1 shows that spectra of the data contain a pervasive and substantial noncentral $\chi^{2}$ component over parts of the $400-$ to $4,000-\mu \mathrm{Hz}$ bands. Section 4.2 provides a more detailed investigation of the data by analyzing spectra and offset coherences. Section 4.3 is a discussion of the results, and section 5 is the conclusions. A supporting information document contains further details and figures to supplement the text, and the pressure data are also provided. 


\section{Data Analysis Methods}

Assuming that a time sequence $x_{t}$ is from a harmonizable random process (i.e., a process that is representable as the superposition of random, infinitesimal harmonic oscillators) implies a spectral representation (Cramér, 1940)

$$
x_{t}=\int_{-1 / 2}^{1 / 2} e^{i 2 \pi \xi t} d X(\xi)
$$

where $E[d X(f)]=0, E$ denotes the expected value and $d X(f)$ is an unobservable increments process, or generalized Fourier transform, whose statistical moments are of interest. If the process is stationary, the increments are orthogonal, so that at two distinct frequencies

$$
E\left[d X\left(f_{1}\right) d X^{*}\left(f_{2}\right)\right]=S\left(f_{1}\right) \delta\left(f_{1}-f_{2}\right) d f_{1} d f_{2}
$$

where $S(f)$ is the true power spectral density and $\delta(x)$ is the Dirac delta function. In other words, stationarity requires that the spectral density at distinct frequencies be uncorrelated.

When a process is nonstationary, the spectral representation has nonorthogonal increments, and (2) is replaced by

$$
\mathrm{E}\left[d X\left(f_{1}\right) d X^{*}\left(f_{2}\right)\right]=S_{L}\left(f_{1}, f_{2}\right) d f_{1} d f_{2}
$$

where $S_{L}$ is the Loève spectrum (Hanssen et al., 2004; Loève, 1945, 1946). Nonstationarity implies that the spectral density at distinct frequencies will be correlated. In the presence of forcing at a given frequency, this also implies that power will be redistributed from that point to other frequencies. Since such a power transfer cannot be modeled as a linear process, this means that forced nonstationarity is inherently nonlinear. Further, it is well known that while linear processes are essentially Gaussian (Mallows, 1967), nonlinear processes can lead to non-Gaussian behavior. These properties will be exploited to understand seafloor pressure data in the IGW band.

The fundamental unit of spectral frequency resolution is the Rayleigh denoted by $R=1 / N \Delta$, where $N$ is the time series length and $\Delta$ is the sample interval. For the 60-day analysis intervals used widely in this paper, $R \simeq 0.19 \mu \mathrm{Hz}$.

\section{Data}

The data used in this study were collected as a component of the Hawaii Ocean Mixing Experiment (HOME) in 2001-2002; see Pinkel et al. (2000) for details about HOME. The specific time series is from site PN2 located at $26^{\circ} 52.5^{\prime} \mathrm{N}, 161^{\circ} 56.7^{\prime} \mathrm{W}$, north-northwest of the island of Kauai at 5,235-m water depth. The data were sampled at $64 / \mathrm{hr}$, and the record length is 389 days beginning in late April 2001. The pressure sensor is a multiturn Bourdon tube with an optical readout, as described by Filloux (1971, 1980, 1983). The instrument was calibrated against a manometer in the laboratory before and after deployment.

A large-amplitude ( $\sim 300$ Pa peak to peak) event is present in the time series at $\sim 62$ days from the start of the record that is due to a tsunami generated by a M8.4 earthquake offshore Peru. This biased many of the analyses in this paper, and so a 4-day-long section beginning at the time of tsunami arrival was removed from the data set for all analyses save those based on the entire record. The remaining five seismic events during the measurement interval with magnitudes above M7 were searched for in the time series, but only a M7.8 event in China in mid-November 2001 could be detected, and even then was barely discernible.

Figure 1 shows a multitaper power spectrum of the entire pressure record. The time-bandwidth $N W$ of the estimate is 8 with 15 Slepian tapers, so the resolution bandwidth is $0.48 \mu \mathrm{Hz}$ and there are about 30 degrees of freedom at each frequency. The diurnal, semidiurnal, and overtides are readily apparent at frequencies below $\sim 100 \mu \mathrm{Hz}$. The spectrum is approximately proportional to $1 / f^{2}$ to $\sim 1,800 \mu \mathrm{Hz}$, beyond which it flattens and becomes humped due to the rising influence of swell-generated IGWs with increasing frequency, and finally falls off beyond $\sim 8,000 \mu \mathrm{Hz}$ due to hydrodynamic filtering of free IGWs as their wavelength decreases toward the water depth. In shallow water, the spectrum remains flat or grows, depending on the strength of 


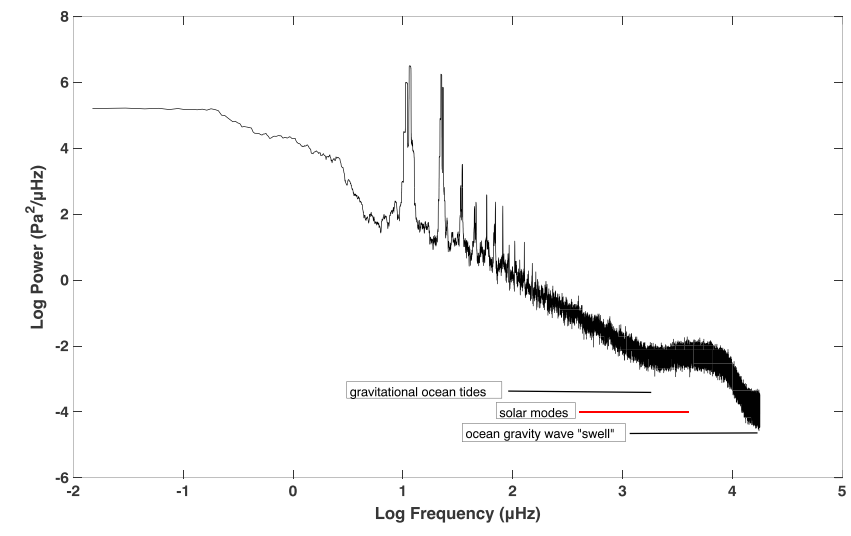

Figure 1. The multitaper power spectral density of the entire bottom pressure record versus frequency on a log-log scale. The time-bandwidth is 8 , and 15 Slepian tapers were applied, yielding 30 degrees of freedom per frequency and a resolution bandwidth of $0.48 \mu \mathrm{Hz}$. The horizontal red line shows the analysis band of $400-4,000 \mu \mathrm{Hz}$ used in this paper; the line also denotes the instrument noise level. Also shown are the approximate frequency bands in which two other energy sources for infragravity waves have been identified or postulated. trapped edge waves, until it reaches the swell band at $\sim 40,000 \mu \mathrm{Hz}$. Figure 1 is similar to spectra for other deep ocean pressure records (e.g., Filloux, 1980; Godin et al., 2013; Webb et al., 1991). Figure 1 also shows the bands where proposed mechanisms for IGWs occur, and whose investigation is a key goal of this paper. The buoyancy frequency at the location of the instrument is less than $200 \mu \mathrm{Hz}$, so that internal waves, which propagate at frequencies below the buoyancy frequency do not contribute to the pressure variance in the 400 - to $4,000-\mu \mathrm{Hz}$ bands.

The instrument noise level of $\sim 10^{-4} \mathrm{~Pa}^{2} / \mu \mathrm{Hz}$ is reached near the Nyquist frequency in Figure 1. The horizontal red line denotes the 400 - to $4,000-\mu \mathrm{Hz}$ analysis band of this paper as well as the noise level. It is important to note that the spectral level over the analysis band is 1 to 2 orders of magnitude above the instrument noise level. What is less apparent is that the variability of the high-frequency components of the spectrum is larger than expected for a $\chi_{30}^{2}$ variate. Figure 2 shows the power spectrum detail over just the $400-$ to $4,000-\mu \mathrm{Hz}$ band, which suggests that the variability rises with frequency.

A set of tidal components comprising 6 long period, 21 diurnal, 19 semidiurnal, and 22 overtides was fit to the entire record using a robust iterative least squares algorithm (Leffler \& Jay, 2009) that was extensively tested on real and synthetic bottom pressure data to confirm the optimal Cauchy weighting function. The resulting constituents are shown in Table S1 in the supporting information for reference. As is typical, the pressure data are dominated by the diurnal and semidiurnal tides, with the K1, M2, O1, and S2 components having amplitudes of 1,600; 1,261; 929; and $761 \mathrm{~Pa}$, respectively.

Text S3 and Figure S4 in the supporting information describe the coherence between the PN2 pressure record and a second one located $236-\mathrm{km}$ distant. The coherence is fit well by a model of random waves propagating in a horizontal plane with different directions, but with similar magnitudes and the same phase velocity for a given frequency, as originally derived by Aki (1957); see also Webb (1986). This clearly establishes the presence of free, isotropic, horizontally propagating IGWs over the analysis frequency band.

\section{Results}

\subsection{Noncentrality in the Pressure Spectrum}

For the remainder of this paper, power spectra are obtained on data that are high-pass filtered by twice passing a three-pole Butterworth filter with a 3-dB point at $46 \mu \mathrm{Hz}$ forward and backward across them. The multitaper spectrum of a 60-day section of the high-passed pressure record is computed with a time-bandwidth of 5 and 9 Slepian tapers, yielding a resolution bandwidth of $1.9 \mu \mathrm{Hz}, 18$ degrees of freedom per frequency, and sidelobe protection of at least $120 \mathrm{~dB}$. The log spectrum versus log frequency over the $200-$ to $4,000-\mu \mathrm{Hz}$ bands is then detrended using a quadratic polynomial to yield a standardized spectrum. The result over a selected analysis band is plotted as linear frequency against linear standardized spectrum. Linear scale spectra are used because they enable resolution of the bandwidth of spectral features, and are easily assessed for significance because the standard deviation of a multitaper estimate is the noise level divided by the square root of the number of Slepian sequences used to obtain it. Figure S1 shows the standardized power spectrum of the entire pressure record over two frequency intervals and the degrees of 3.6 freedom over 400-4,000 $\mu \mathrm{Hz}$.

Standardized spectra for 60-day long, 50\% overlapping blocks were computed. A possible cause for higher than expected spectral variability (see Figure 2) is the presence of deterministic components that would be 


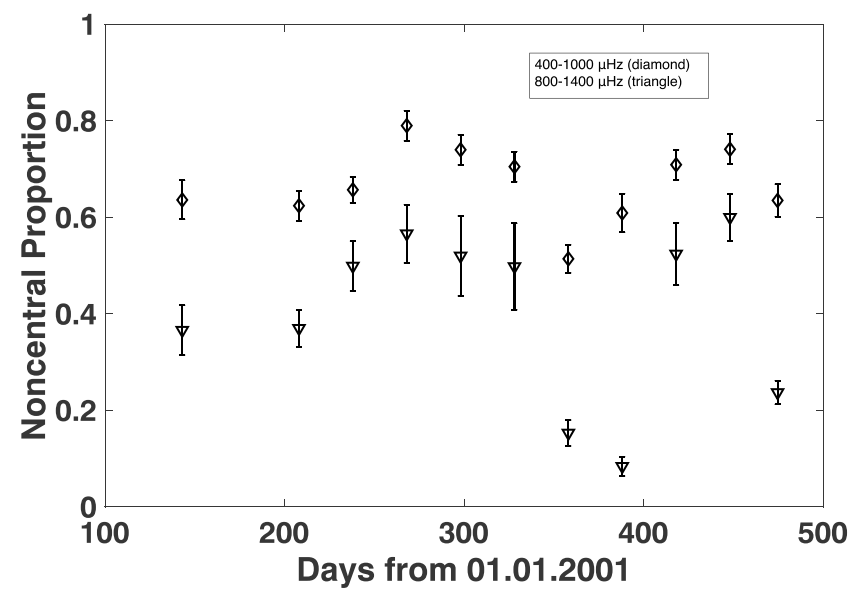

Figure 3. The proportion of the spectral estimates that are consistent with a noncentral $\chi^{2}$ distribution after fitting the mixture model (4) over the frequency bands defined in the inset box against year-day. The symbols denote the maximum likelihood estimate, and the error bars are double-sided 95\% confidence limits using the asymptotic Fisher information for the maximum likelihood estimate. The mixture model was fit to 60-day time segments overlapped by $50 \%$ except for the first one.

distributed as the noncentral $\chi^{2}$ distribution that has longer right tails than its central counterpart. In this context, deterministic means a process whose bandwidth is smaller than or comparable to the Rayleigh resolution and whose phase remains substantially constant over the analysis interval. A mixture noncentral and central $\chi^{2}$ model

$$
2 K\left[\varepsilon \chi_{2 K}^{2}(\lambda)+(1-\varepsilon) \chi_{2 K}^{2}(0)\right]
$$

where $\varepsilon$ is the noncentral proportion and $\lambda$ is the noncentrality parameter, was fit to each data block over a range of frequency bands by the maximum likelihood method implemented using a nonlinear multivariable programming solver.

Figures 3 and 4 show the noncentral proportion over the 400- to 1,000and $800-$ to $1,400-\mu \mathrm{Hz}$ bands and the 2,000- to $3,000-; 2,500-$ to 3,500 ; and 3,000- to $4,000-\mu \mathrm{Hz}$ bands, respectively. The mixture model could not be fit to the frequency bands spanning the values in Figures 3 and 4, where typical solutions are either approximately central $\chi_{18}^{2}$ or yield a nonphysical negative mixture proportion because the empirical distributions of the spectra are shifted to the left compared to a central $\chi_{18}^{2}$ distribution.

Over the lower frequency bands (Figure 3), there is evidence for a weak semiannual variation peaking in the fall and spring, although the minimum in winter is quite pronounced for the $800-$ to $1,400-\mu \mathrm{Hz}$ bands. However, the statistical procedure is not robust, and the anomalously low values over $800-1,400 \mu \mathrm{Hz}$ have noncentrality parameters that are about twice as large as the remaining ones, possibly due to the signature of frequent winter storms in the data. The noncentrality parameters are nearly constant over $400-1,000 \mu \mathrm{Hz}$. The noncentral proportion averages (standard deviations) are $0.669(0.074)$ and $0.402(0.167)$ for the 400- to $1,000-$ and $800-$ to $1,400-\mu \mathrm{Hz}$ bands, respectively.

For the higher frequency bands (Figure 4), the evidence for seasonality is weaker. The average (standard deviation) for the noncentral proportion is 0.060 (0.053), 0.285 (0.199), and $0.306(0.053)$ for the 2,000- to $3,000-; 2,500-$ to $3,500-;$ and $3,000-$ to $4,000-\mu \mathrm{Hz}$ bands, respectively. The noncentral proportion is barely distinguishable from zero in the lowest frequency band, and the standard deviation for the middle frequency band is large because of the high values observed in summer 2001.

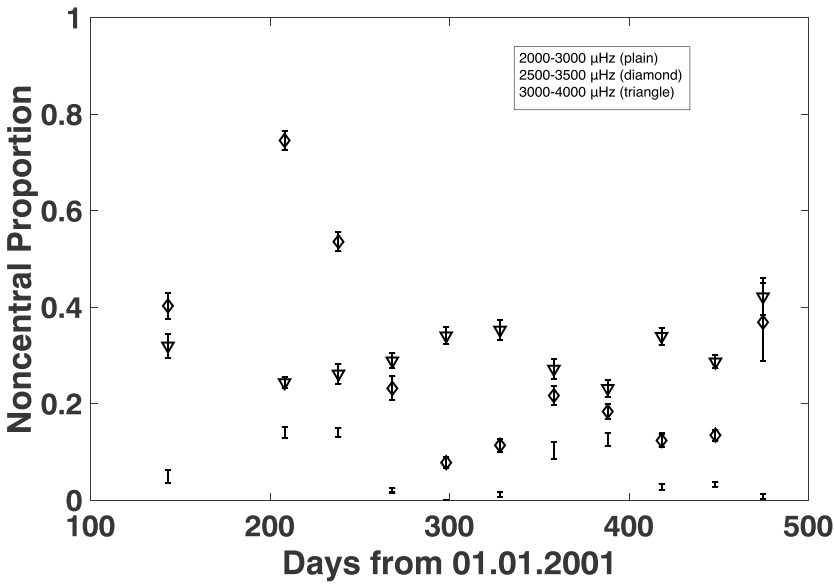

Figure 4. The proportion of the spectral estimates that is consistent with a noncentral $\chi^{2}$ distribution after fitting the mixture model (4) over the frequency bands defined in the inset box against year-day. The symbols denote the maximum likelihood estimate, and the error bars are double-sided 95\% confidence limits using the asymptotic Fisher information for the maximum likelihood estimate. The mixture model was fit to 60-day time segments overlapped by $50 \%$ except for the first one.
Figure S2 contains four examples of the mixture $\chi^{2}$ model fit that are typical rather than unusual. The empirical distribution of a set of spectral estimates is obtained using a kernel density estimator, which is the sum of smoothing operators applied repeatedly across the support and is in effect a smoothed probability histogram (Chave, 2017, section 4.8.3). In all instances, the fit of the mixture $\chi^{2}$ model is qualitatively reasonable.

Overall, the picture that emerges is one of narrowband elements comprising over half of the spectral variability over $400-1,000 \mu \mathrm{Hz}$, decreasing with increasing frequency, an anomalous (i.e., not central $\chi^{2}$ ) distribution over $1,400-2,000 \mu \mathrm{Hz}$ and the reemergence of a narrowband element as frequency rises above $2,000 \mu \mathrm{Hz}$, but with a noncentral proportion of about one third. The first and last of these bands correspond to regions of enhanced power in Figure S1 (middle panel), while the intermediate band where the $\chi^{2}$ mixture model fails is at a relative minimum in power.

Luther et al. (2014) investigated the same pressure record and suggested that over the $\sim 140$ - to $1,100-\mu \mathrm{Hz}$ bands, IGWs may be forced by tides. Figure $\mathrm{S} 1$ (middle panel) suggests that there is a region of enhanced power over the interval $\sim 400-1,400 \mu \mathrm{Hz}$, and the bottom panel shows that this is not distributed uniformly in frequency, but rather is higher in the vicinity 
Table 1

Variance in Tides/Total Variance Over $400-1,000 \mu \mathrm{Hz}$

\begin{tabular}{|c|c|c|c|c|}
\hline Year-day & $\begin{array}{c}\sigma_{\text {line }}^{2} \\
0.99(0.95)\end{array}$ & $\sigma_{\text {total }}^{2}$ & $\begin{array}{c}\text { Tidal fraction } \\
0.99(0.95)\end{array}$ & $\begin{array}{c}\text { Noncentral } \chi^{2} \\
\text { fraction }\end{array}$ \\
\hline 143 & $1.96(5.59)$ & 16.22 & $0.121(0.345)$ & 0.636 \\
\hline 208 & $2.25(7.33)$ & 23.09 & $0.097(0.318)$ & 0.624 \\
\hline 238 & $2.04(6.83)$ & 20.03 & $0.102(0.341)$ & 0.657 \\
\hline 268 & $1.95(5.63)$ & 16.57 & $0.118(0.340)$ & 0.790 \\
\hline 298 & $1.63(5.30)$ & 16.07 & $0.101(0.330)$ & 0.740 \\
\hline 328 & $2.16(6.34)$ & 18.24 & $0.118(0.348)$ & 0.705 \\
\hline 358 & $2.11(6.50)$ & 19.40 & $0.109(0.335)$ & 0.514 \\
\hline 388 & $1.84(5.09)$ & 17.18 & $0.107(0.296)$ & 0.609 \\
\hline 418 & $1.74(5.33)$ & 14.28 & $0.122(0.373)$ & 0.709 \\
\hline 448 & $1.41(4.70)$ & 15.15 & $0.093(0.310)$ & 0.741 \\
\hline 475 & $1.63(5.45)$ & 15.77 & $0.103(0.346)$ & 0.635 \\
\hline
\end{tabular}

of $600 ; 760 ; 950 ; 1,070 ; 1,200$; and $1,350 \mu \mathrm{Hz}$ than at intervening frequencies. A multitaper $F$ test was applied to 60 -day long, adjoining data segments, and all frequencies where the $F$ statistic was significant at the 0.99 level were compared to the harmonics of the tidal frequencies from Table S1. At nearly all frequencies where the $F$ test was significant, a tidal harmonic exists within $2 R$, and more typically within the $1 R$ resolution of the $F$ test, and these occur every $6-9 \mu \mathrm{Hz}$ on average. Figure S3 shows the frequencies of the significant $F$ statistics that occur within $0-1 R$ and $1-2 R$ of a tidal harmonic. These are not evenly distributed in frequency or time but tend to be concentrated toward the lower end of the $400-$ to $1,400-\mu \mathrm{Hz}$ bands and with some correlation to Figure S1 (bottom panel), which is an average over the 1-year record. In addition, significant $F$ tests that do not correlate with the frequencies of tidal harmonics occur more prevalently over $800-1,400 \mu \mathrm{Hz}$ as compared to $400-1,000 \mu \mathrm{Hz}$. Finally, the multitaper $F$ test applied to the entire pressure record does not yield very many significant values at tidal harmonics, suggesting that they are intermittent rather than persistent. This ephemeral characteristic is consistent with the hypothesized forcing mechanism of variable nonlinear amplification of the barotropic tide in the shallow seas around the Pacific Ocean, especially the Gulf of Alaska. The variability arises due to nontidal sea level fluctuations and coastal currents, followed by propagation off the shallow seas and into deep water with refraction and diffraction from multiple sources that this implies.

Since multitaper $F$ tests that are significant at the 0.99 level occur predominantly at tidal harmonics over $400-1,000 \mu \mathrm{Hz}$, an estimate for their variance obtains from reshaping the multitaper power spectrum at those frequencies, thereby separating the tidal harmonics from the stochastic power spectrum. This was carried out over 60 day, 50\% overlapped data sections. Table 1 contains the center year-day of each data section relative to 01.01.2001, the total variance of tidal harmonics, the variance in the original spectrum obtained by integration over frequency, the ratio of the line to spectrum variance, and the noncentral fraction from Figure 4. For comparison purposes, Table 1 also shows the tidal variance and tidal fraction obtained by reshaping at the 0.95 level. The variance ratio is $\sim 15-20 \%$ of the noncentral fraction using a 0.99 criterion in each case and is a lower bound because this calculation does not account for tidal harmonics whose $F$ tests are below the 0.99 level. Using a 0.95 reshaping criterion, the tidal harmonics are $\sim 40-50 \%$ of the noncentral fraction. Consequently, an important source of noncentrality in these pressure data is tidal harmonics over $400-1,000 \mu \mathrm{Hz}$, but an additional contribution exists that remains to be explained.

\subsection{Power Spectra and Offset Coherences}

Text S2 in supporting information provides a summary of the characteristics of solar normal modes. Some key properties are a planetary scale, $Q$ s of several thousand that rise with increasing frequency, which leads to the persistence of a given mode for about 60 days on average, amplitudes that are random and nonstationary, and the expectation that a given mode will be observed on Earth about one sixth of the time or conversely that about one sixth of the modes in a given time segment will be active.

This section contains a more detailed look at the frequency domain properties of the seafloor pressure record using two tools: standardized (as defined in section 4.1) power spectra and offset coherences defined in Text S1. The choice of 60-day data sections is motivated by the desire for a resolution bandwidth that can detect high $Q$ features and the persistence time of p-modes. From Figure 1, the power spectral density is at least an order of magnitude above the noise level over $400-4,000 \mu \mathrm{Hz}$, and so a conservative estimate of the noise level is the 0.1 quantile over an analysis band. This is larger than the 0.05 quantile used in Thomson and Vernon (2015) and likely overestimates the noise level for frequencies below $\sim 1,500 \mu \mathrm{Hz}$ (see Figure 1). The figures presented here and in the supporting information are examplars rather than extreme, and many other time segments or frequency bands could have been depicted, consistent with the one sixth occurrence rate.

The coherence is the generalized likelihood ratio test for independence (Schreier \& Scharf, 2010, section 4.5.3), either between time series or between frequencies within a single time series. In addition, a 
multitaper offset coherence has the resolution bandwidth $2 W$ on the ordinary frequency axis, but the Rayleigh resolution $R$ on the offset frequency axis (Thomson, 2001). Plotting of the offset coherence is carried out as in Thomson and Vernon (2015, section 9) to emphasize the most highly coherent elements in the data. Since a key goal is evaluation of the null hypothesis that the pressure spectra are independent of solar modes, the offset coherence will be evaluated under the condition that its true value is zero, implying no nonstationarity. The probability density function for coherence is given in Thomson and Chave (1991, equation 2.53; note that the factor $m-1$ at the lead of that equation should be in the numerator), and when the true coherence is zero, reduces to

$$
p\left(\widehat{\gamma}^{2}\right)=(K-1)\left(1-\widehat{\gamma}^{2}\right)^{K-2}
$$

where $K$ is the number of Slepian tapers used in the multitaper estimator. Integrating (5) over 0 to $x$, where $x \leq 1$, yields the cumulative probability distribution (cdf)

$$
P(x)=1-(1-x)^{K-1}
$$

The offset coherence is plotted as

$$
-\log _{10}(1-P)=-(K-1) \log _{10}\left(1-\widehat{\gamma}^{2}\right)
$$

and is clipped at a value of 3 to 5 . Values of 1, 2, 3, 4, and 5 for (7) correspond to the $0.9,0.99,0.999,0.9999$, and 0.99999 probability levels that two frequencies are correlated.

For reasons that will become apparent, the presentation will extend from high to low frequencies. Figure 5 shows the standardized power spectrum over 3,700-3,750 $\mu \mathrm{Hz}$ (central period of $\sim 268 \mathrm{~s}$ ) for a 60 -day data block ranging from year-day 418-478, or early spring 2002 . The solid horizontal line shows the estimated noise level, and the horizontal dotted lines show successive increments in standard deviations from that value. The vertical dashed lines show the nominal center frequencies of solar p-modes from MDI (Rhodes et al., 1997) close to the peaks in the spectrum, without including rotational splitting. Given the high degree of these modes, the latter would nearly cover the figure with vertical dashed lines.

There are two peaks in Figure 5 that exceed 11 standard deviations from the 0.1 quantile, and an additional peak that exceeds 9 standard deviations. The probability of a single peak exceeding $9 \sigma$ above the 0.1 quantile is $3.2 \times 10^{-7}$ for a central $\chi_{18}^{2}$ variate. Consequently, the probability of independently observing three such peaks is $3.3 \times 10^{-20}$, which is effectively impossible unless there is a nonrandom component present. The probability of a single peak exceeding $11 \sigma$ above the 0.1 quantile is $3.0 \times 10^{-9}$, so the probability of independently observing two such peaks is $9.0 \times 10^{-18}$, which is also extremely unlikely. This strongly argues for the presence of quasi-harmonic forcing at one or more frequencies in the analysis band.

Figure 5 also shows the center frequencies of the ${ }_{8} \mathrm{~S}_{4},{ }_{4} \mathrm{~S}_{9},{ }_{0} S_{29}$, and ${ }_{6} \mathrm{~S}_{8}$ terrestrial normal modes given by Masters and Widmer (1995) as labeled vertical dash-dotted lines. Both ${ }_{8} \mathrm{~S}_{4}$ and ${ }_{0} \mathrm{~S}_{29}$ are located near large spectral peaks. Masters and Widmer (1995) give a model frequency and $Q$ of $3707.73 \mu \mathrm{Hz}$ and 93.5 for the former; a terrestrial normal mode peak will have a Lorentzian shape, and with this $Q$, the full bandwidth at half power would be $\sim 40 \mu \mathrm{Hz}$ if the mode were present, which is clearly not observed. Smith and Masters (1989) give a measured frequency of $3,724.75 \mu \mathrm{Hz}$ and a $Q$ of 222 for ${ }_{0} S_{29}$. With the specified $Q$, the full bandwidth at half power would be $16.8 \mu \mathrm{Hz}$. However, the bandwidth of the peak in Figure 5 is only $3 \mu \mathrm{Hz}$, hence is not consistent with a terrestrial mode origin.

The offset coherence was computed over $3,700-3,750 \mu \mathrm{Hz}$ and for offset frequencies up to $\pm 25 \mu \mathrm{Hz}$. After excluding a $2 R$ wide band centered at zero offset frequency, the cumulative distribution function (6) was computed from the coherence estimates. Under the probability integral transform, the coherence cdf estimates should have a uniform distribution if there is no correlation between frequencies, and hence a percent-percent plot (Chave, 2017, section 4.8.4) of the quantiles of the uniform distribution against the sorted cdf estimates should plot as a straight line. Figure 6 shows such a plot for the offset coherence, and there is no need for a formal test to establish that there is significant coherence between frequencies, and hence nonstationarity, in these pressure data. Percent-percent plots over other frequency bands are similar to Figure 6 and will not be shown. 


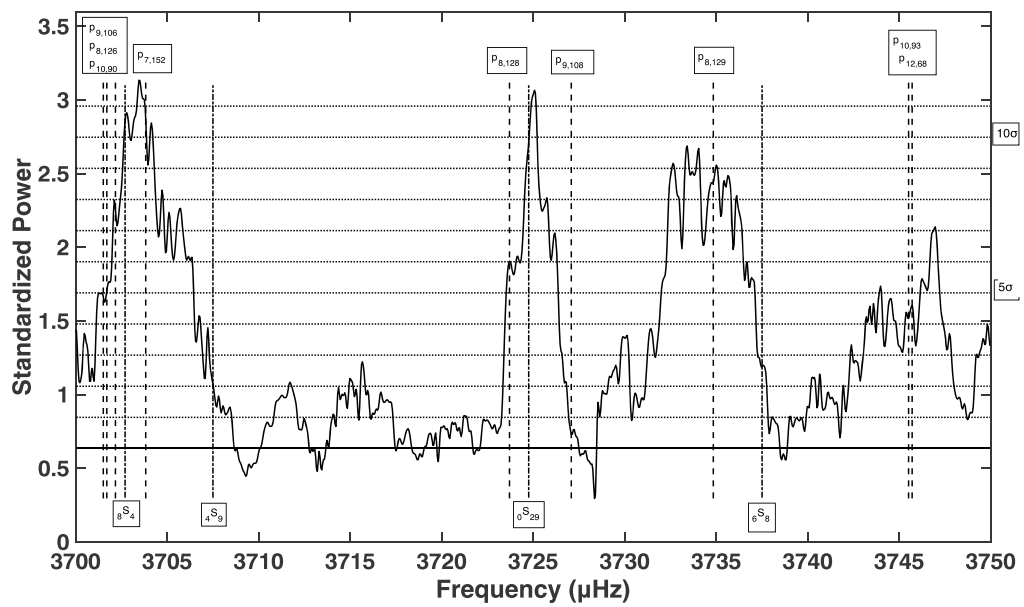

Figure 5. Standardized pressure power spectral density versus frequency on linear scales over year-days $418-478$. The time-bandwidth is 5, and there are nine data tapers, yielding a resolution bandwidth of $1.9 \mu \mathrm{Hz}$ and 18 degrees of freedom per frequency. The solid horizontal line is the 0.1 quantile of power over the $3,700-$ to $3,750-\mu \mathrm{Hz}$ bands that defines the noise level, and the dotted horizontal lines are increments of 1 standard deviation from that value, with the 5 and $10 \sigma$ levels labeled on the right. The vertical dashed lines show the locations of solar p-modes, excluding rotational splitting, as listed at the top, and the vertical dash-dotted lines are the locations of terrestrial spheroidal normal modes labeled at the bottom.

Figure 7 shows the transformed offset coherence using (7) along with its color map. The abscissa is the frequency ranging from 3,700 to $3750 \mu \mathrm{Hz}$, and the ordinate is the offset frequency ranging from -25 to $+25 \mu \mathrm{Hz}$. Excluding a $2 R$ wide band centered at zero offset frequency, there are 14,973 $(2,423)$ frequencyoffset frequency pairs above 4 (5), and these appear in horizontally elongate clusters that reflect the different resolutions in ordinary frequency and offset frequency. Figure 7 is quite striking in that symmetric trapezoidal blocks appear about zero offset frequency at each of the broad peaks in Figure 5. The trapezoidal shape is not difficult to understand; if frequency $f_{1}$ is coherent with frequency $f_{2}>f_{1}$, then the coherence will be high at a positive offset frequency $f_{2}-f_{1}$ at frequency $f_{1}$, and hence at a negative offset frequency $f_{1}-f_{2}$ at frequency $f_{2}$. Each of the trapezoidal blocks along zero offset frequency has an internal structure that suggests that each of the peaks in Figure 5 comprises numerous narrowband components that are themselves coherent. In fact, the spacing of the elongate peaks within each block is approximately the $\sim 440 \mathrm{nHz}$ p-mode rotational splitting frequency (see Text S2). Trapezoidal blocks also occur at offset frequencies consistent with coherence between the peaks, which coherence certainly extends beyond the $\pm 25 \mu \mathrm{Hz}$ offset frequencies

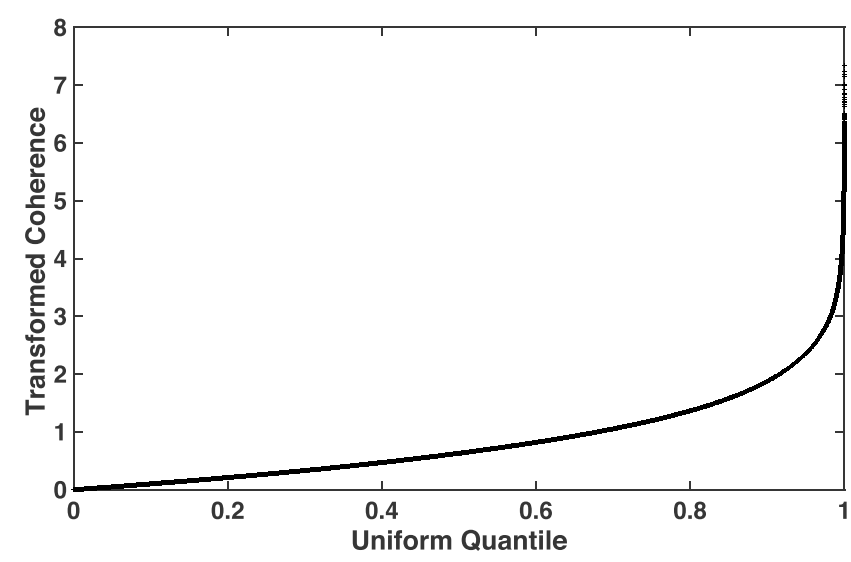

Figure 6. Percent-percent plot showing the ranked transformed coherences using (6) plotted against uniform quantiles. The result would be a straight line if the hypothesis that there is no interfrequency correlation in the pressure data were correct. shown in the figure. The picture that emerges is one of strongly nonstationary behavior that is manifest both as high offset coherence within spectral peaks and between them.

Text S4 describes Figures S5 and S6 that show the power spectrum and offset coherence over $2,800-2,870 \mu \mathrm{Hz}$ (central period of $\sim 353 \mathrm{~s}$ ) for a 60 -day data block ranging from year-day 418-478. These demonstrate the presence of numerous peaks at the resolution bandwidth that display marked nonstationarity. The occurrence of peaks near the center (azimuthal quantum number $m$ of zero) frequency of many solar p-modes is suggestive of their origin.

Figure 8 shows the power spectrum over the frequency band 1,745$1,795 \mu \mathrm{Hz}$ (central period of $565 \mathrm{~s}$ ) for year-days 358-418 or winter 2002. The largest peak in the figure is $11 \sigma$ above the noise level, followed by a $9 \sigma$ peak, a $7 \sigma$ peak, and two $4 \sigma$ ones. The probability of independently observing the two largest peaks in a central $\chi_{18}^{2}$ population is $9.9 \times 10^{-16}$, and for the three largest peaks it is $2.7 \times 10^{-20}$. Either of these scenarios is extremely unlikely, and so the presence of a nonrandom component in the pressure data is assured. The three largest peaks in Figure 8 


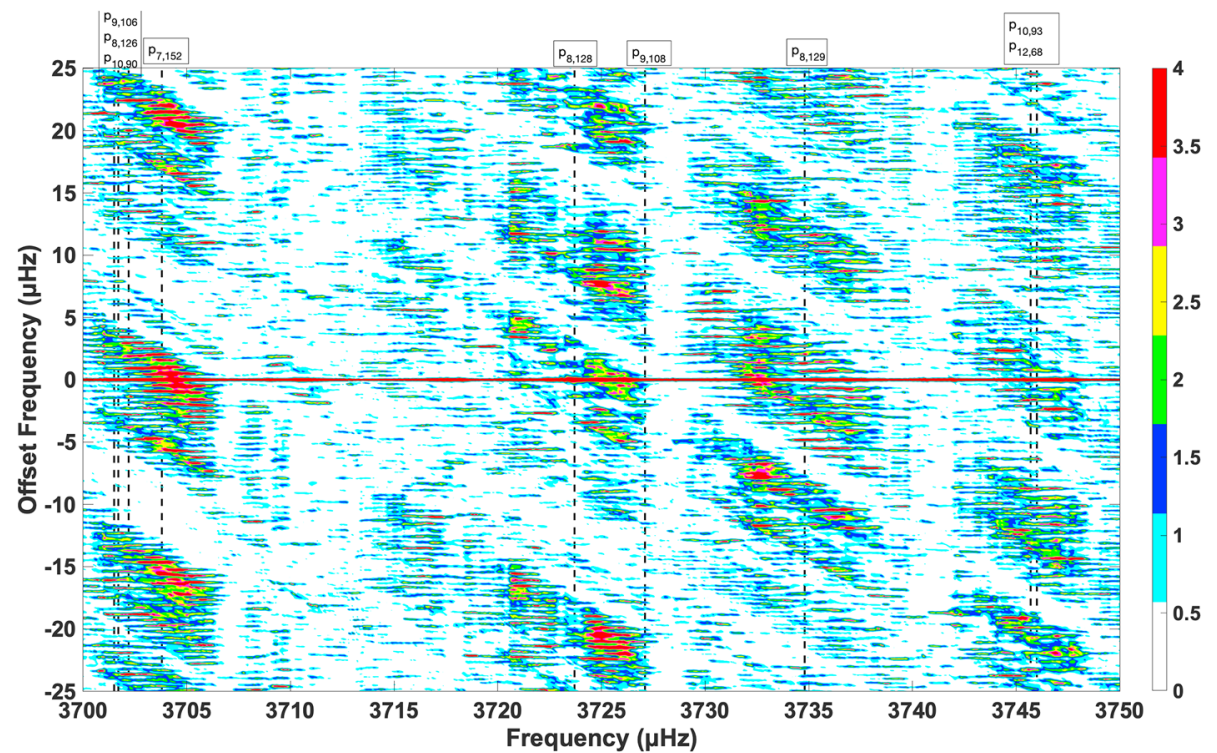

Figure 7. Contours of the offset coherence transformed using (7) against frequency on the abscissa and offset frequency on the ordinate over 3,700-3,750 $\mu \mathrm{Hz}$ for year-days $418-478$. The time-bandwidth is 5 , and nine Slepian tapers were used, yielding 18 degrees of freedom per frequency. The vertical dashed lines denote the center frequencies of the p-modes delineated at the top.

(as well as the smaller ones) have a full bandwidth at half power of $\sim 2 \mu \mathrm{Hz}$, implying a $Q$ of at least 890 . It is notable that most of the peaks in Figure 8 occur near the center $(m=0)$ frequency of solar p-modes.

Figure 9 contains the offset coherence over the same band and data interval as in Figure 8; note the different color map as compared to Figure 7. There is a very large trapezoidal coherent patch in the middle of the plot extending from $\sim 1,758-1,785 \mu \mathrm{Hz}$ along zero offset frequency, indicating pervasive coherence within the

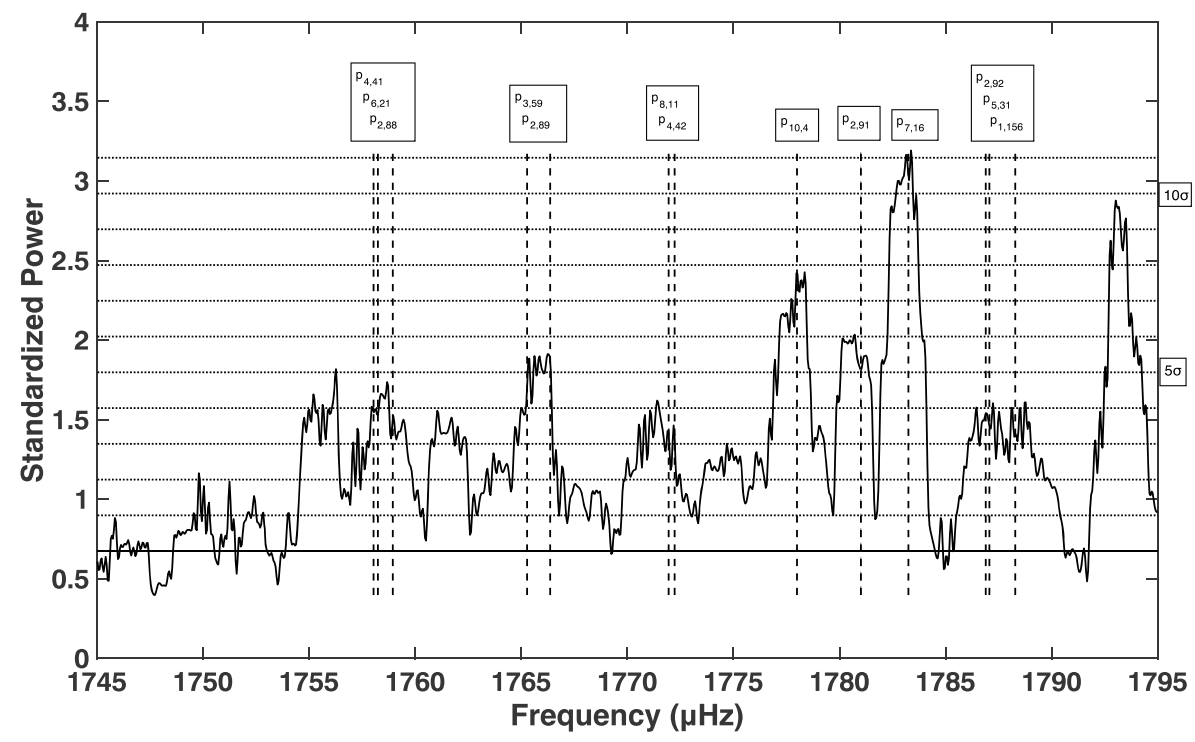

Figure 8. Pressure power spectral density versus frequency on linear scales over year-days 358-418. The time-bandwidth is 5, and there are nine data tapers, yielding a resolution bandwidth of $1.9 \mu \mathrm{Hz}$ and 18 degrees of freedom per frequency. The solid horizontal line is the 0.1 quantile of power over the $1,745-$ to $1,795-\mu \mathrm{Hz}$ bands that defines the noise level, and the dotted horizontal lines are increments of 1 standard deviation from that value, with the 5 and $10 \sigma$ levels labeled at right. The vertical dashed lines show the locations of solar p-modes, excluding rotational splitting, as listed at the top. 


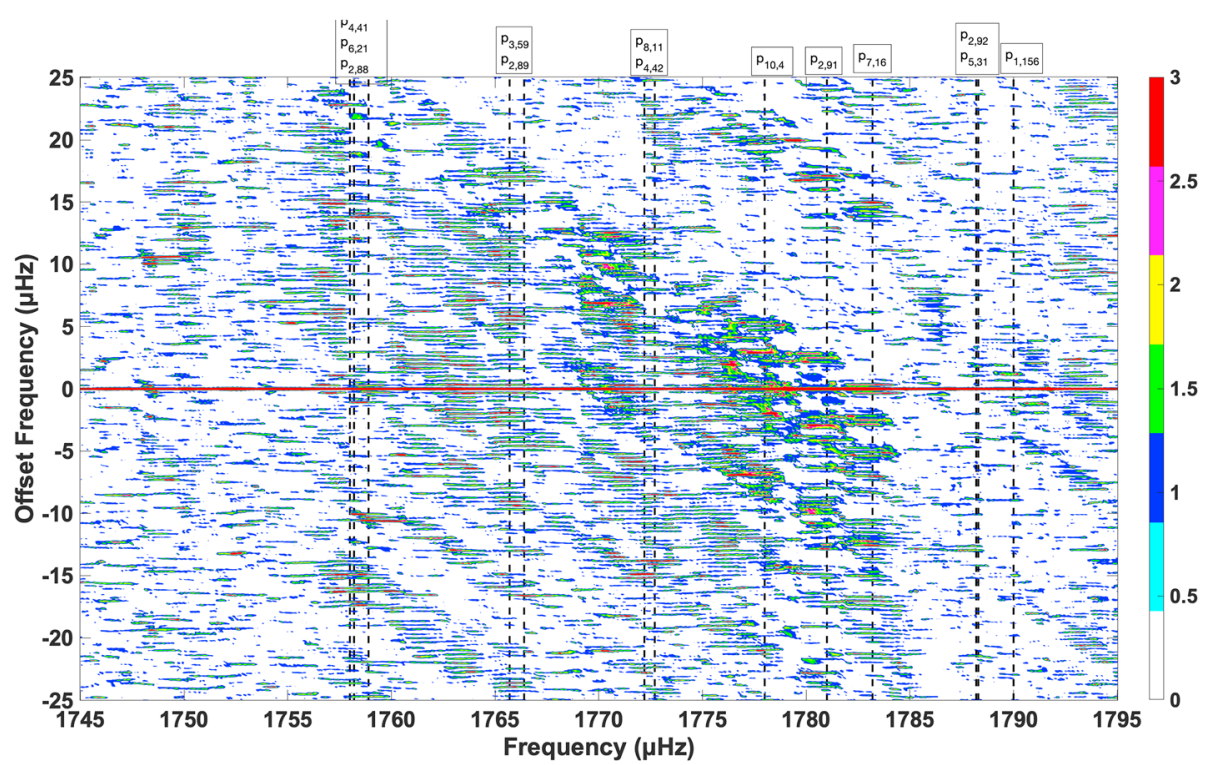

Figure 9. Contours of the offset coherence transformed using (7) against frequency on the abscissa and offset frequency on the ordinate over $1,745-1,795 \mu \mathrm{Hz}$ for year-days $358-418$. The time-bandwidth is 5, and nine Slepian tapers were used, yielding 18 degrees of freedom per frequency. The vertical dashed lines denote the center frequencies of the p-modes delineated at the top. Note the different color scale compared to Figure 7.

spectrum over the middle two thirds of Figure 8. As in Figure 7, there is fine structure within the trapezoidal structure at offset frequency intervals of $\sim 400 \mathrm{nHz}$. There is also coherence with the large peak in Figure 8 at $1,793 \mu \mathrm{Hz}$ and that peak is in turn coherent with something that is $22-24 \mu \mathrm{Hz}$ higher in frequency, as seen through the coherent patch at the upper right of the figure.

Text S5, along with Figures S7 and S8, describes the power spectrum and offset coherence over 1,175-1,225 $\mu \mathrm{Hz}$ for year-days 113-173. These further demonstrate the presence of peaks at the resolution bandwidth with marked nonstationarity, along with the likely presence of tidal harmonics.

Figure 10 shows the multitaper power spectrum over $780-830 \mu \mathrm{Hz}$ for the 60 -day data block ranging from year-day 268-328 or fall 2001. The vertical dashed lines show the nominal frequencies of solar p-modes, including rotational splitting, from Provost et al. (2000). There are five narrow peaks over the $50 \mu \mathrm{Hz}$ analysis band that exceed the noise level by at least $4 \sigma$, and two peaks that exceed it by nearly $6 \sigma$, and all have a bandwidth comparable to the resolution value. Two of the five $4 \sigma$ peaks in Figure 10 occur within $\sim 1 \mu \mathrm{Hz}$ of the $\mathrm{p}_{3,5}$ and $\mathrm{p}_{4,2}$ families of solar normal modes.

The probability of a single peak exceeding $4 \sigma$ above the 0.1 quantile is 0.0097 for a central $\chi_{18}^{2}$ variate. Consequently, the probability of five peaks occurring independently above the $4 \sigma$ level is $8.6 \times 10^{-11}$, which is very unlikely unless there is a nonrandom component present. Further, four of the large peaks in Figure 10 have the characteristic rectangular shape with the resolution bandwidth $2 W$ of narrowband components in multitaper estimates.

The full bandwidth at half power of the peaks in Figure 10 is that of the multitaper spectral window $(1.9 \mu \mathrm{Hz})$, so their $Q$ s are at least 420 , and could be much larger. Figure 10 also shows the location of the ${ }_{0} S_{0}$ terrestrial breathing spheroidal normal mode. Yu et al. (2008) give a frequency of $814.66 \mu \mathrm{Hz}$ and a $Q$ of 5,400 for this mode; this is by far the highest $Q$ terrestrial normal mode. With such a high $Q$, a peak associated with it would have the resolution bandwidth of $1.9 \mu \mathrm{Hz}$. However, there is no peak, but rather a minimum in power, at the frequency of ${ }_{0} S_{0}$.

In a multitaper $F$ test, both $804.1 \mu \mathrm{Hz}$ (second unlabeled peak near the center of Figure 10) and $828.5 \mu \mathrm{Hz}$ (near the right end of Figure 10) are significant at the 0.99 level. The first peak is within $0.7 R$ of the 38th harmonic of tidal constituent $\varepsilon 2$ and $1.3 R$ of the 36th harmonic of tidal constituent H1. The second peak is within $0.1 R$ of the 72nd harmonic of $\pi 1,0.5 R$ of the 37th harmonic of $\mathrm{H} 2,1.3 R$ of the 71st harmonic of $\varphi 1$, and $1.8 R$ of the 77th harmonic of $\mathrm{O} 1$. 


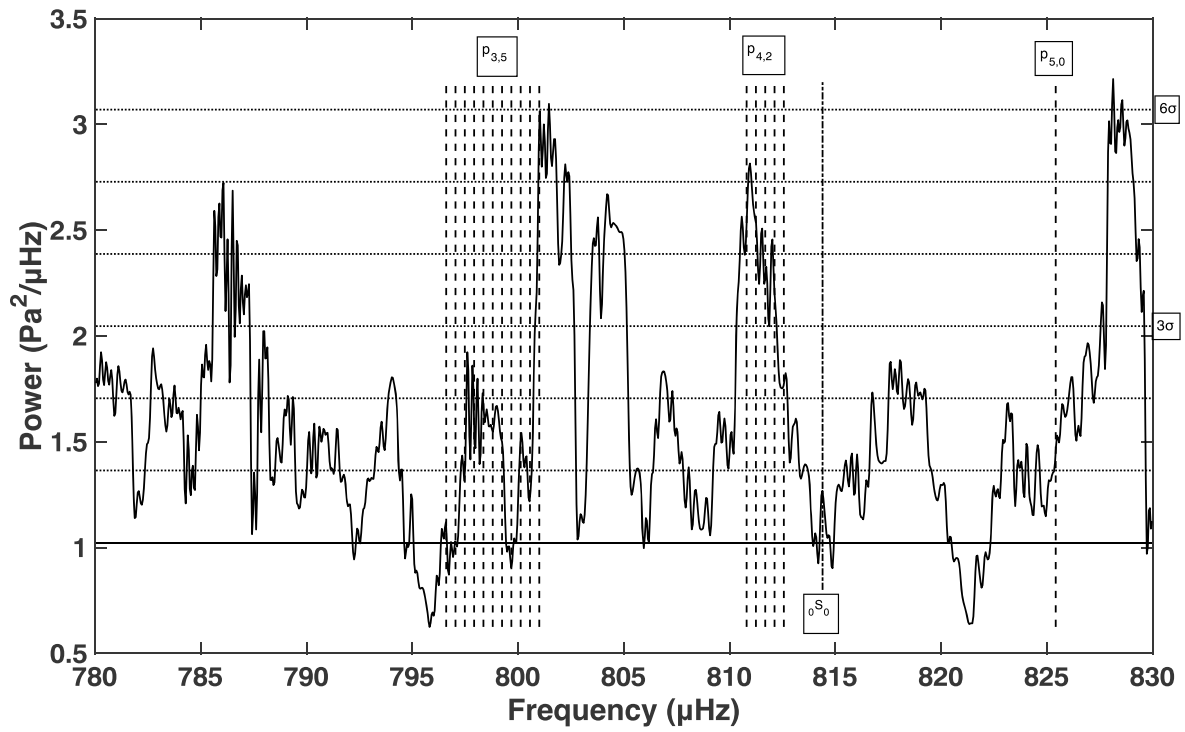

Figure 10. Standardized power spectral density versus frequency on linear scales over year-day 268-328. The time-bandwidth is 5 , and there are nine data tapers, yielding a resolution bandwidth of $1.9 \mu \mathrm{Hz}$ and 18 degrees of freedom per frequency. The solid horizontal line is the 0.1 quantile of power over the 780 - to $830-\mu \mathrm{Hz}$ bands that defines the noise level, and the dotted horizontal lines are increments of 1 standard deviation from that value, with the 3 and $6 \sigma$ labeled at right. The vertical dashed lines show the locations of solar p-modes, including rotational splitting, as listed at the top, and the location of the ${ }_{0} \mathrm{~S}_{0}$ terrestrial spheroidal normal mode is denoted by the dash-dotted line labeled at the bottom.

Figure 11 shows the offset coherence over $780-830 \mu \mathrm{Hz}$ and for offset frequencies of $\pm 25 \mu \mathrm{Hz}$. There are two trapezoidal regions of enhanced offset coherence that have been outlined with red dashed lines. The right trapezoidal pattern over $\sim 808-820 \mu \mathrm{Hz}$ encompasses the peak labeled $\mathrm{p}_{4,2}$ and the $2 \sigma$ peak to its right in Figure 10. The left trapezoidal pattern covering $\sim 782-793 \mu \mathrm{Hz}$ corresponds to the left peak in Figure 10 that does not correspond to any solar p-mode frequency. The two trapezoidal regions are themselves coherent, as seen through the patch of high coherence outlined by dashed ellipses at $-17.5-\mu \mathrm{Hz}$ offset frequency at a frequency of $\sim 809 \mu \mathrm{Hz}$ and at $+17.5-\mu \mathrm{Hz}$ offset frequency at a frequency of $\sim 792 \mu \mathrm{Hz}$. This suggests that there is forcing at $\mathrm{p}_{4,2}$ that is transferred both to the $2 \sigma$ peak to its right and to $\sim 792 \mu \mathrm{Hz}$ by nonstationarity. Note also that neither the location of $\mathrm{p}_{3,5}$ nor the two large peaks to its right at 801.7 and $804.1 \mu \mathrm{Hz}$ nor the $828.5 \mu \mathrm{Hz}$ peak at the right side of Figure 10 display substantial offset coherence, suggesting that they have a different origin that does not involve nonstationarity, consistent with a tidal origin.

\subsection{Discussion}

Solar p-modes are readily detectable throughout the $400-$ to $4,000-\mu \mathrm{Hz}$ analysis band, as seen through the appearance of features with a $Q$ whose lower bound is determined by the resolution bandwidth of the estimate, yet with a value much higher than that of known terrestrial or oceanic phenomena, and in close association with the known frequencies of solar p-modes. This is most readily seen at lower frequencies where the frequency density of p-modes is relatively low. For example, Figures 10 and 11 unambiguously establish the presence of a $5 \sigma$ peak at the frequency of the $\mathrm{p}_{4,2}$ family of modes that is coherent with other local and more distant frequencies. The most probable interpretation of these figures is that power enters the system at some or all of the $\mathrm{p}_{4,2}$ singlets, and then is transferred to other frequencies due to pervasive nonstationarity. Such an interpretation is more ambiguous in Figures 8 and 9 because there are many more families of modes with higher degrees, hence more extensive rotational splitting. The most coherent patch in Figure 8 extends from $\sim 1,777$ to $1,784 \mu \mathrm{Hz}$ at zero offset frequency but extends to lower frequencies as evidenced by the large trapezoidal patch in Figure 9. The largest $(11 \sigma)$ peak at $1,783 \mu \mathrm{Hz}$ in Figure 8 occurs at the center of the $p_{7,16}$ p-mode family but is highly coherent with the $\mathrm{p}_{10,4}$ peak at $1,777 \mu \mathrm{Hz}$, and it is difficult to determine which one is active versus the result of nonstationarity or whether both peaks are the result of solar mode forcing. Similar interpretations can be inferred at higher frequencies, although uncertainty rises with frequency due 


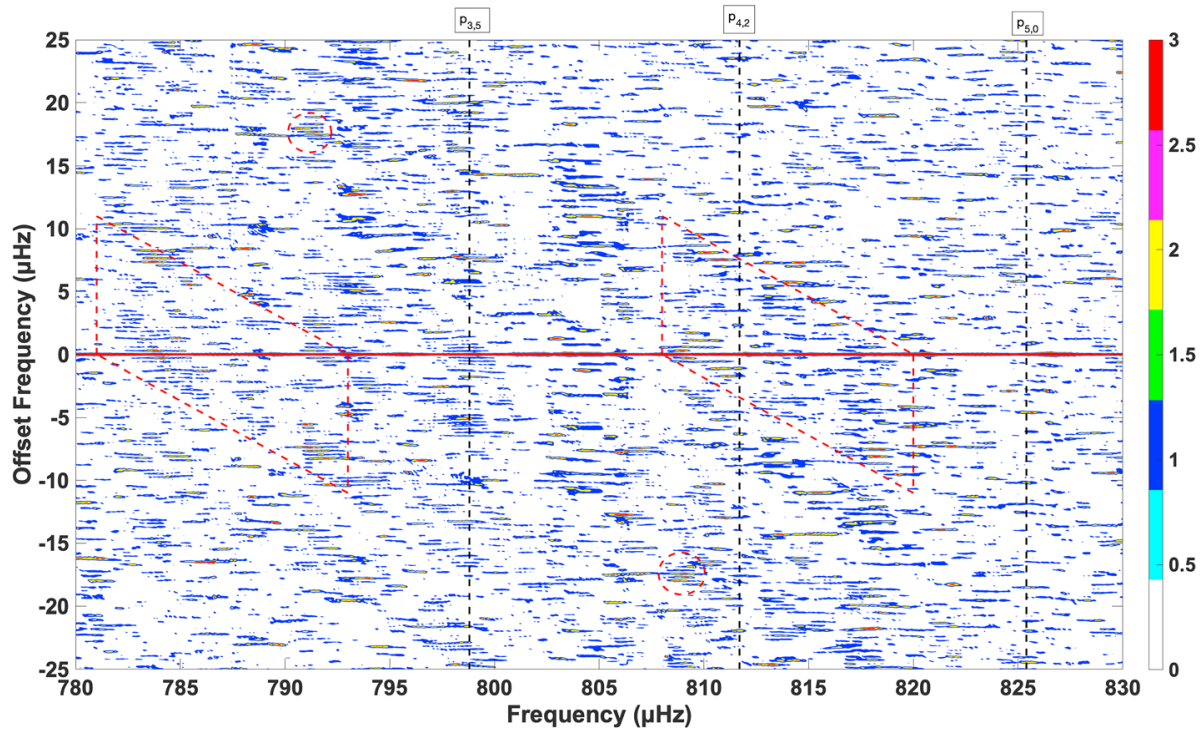

Figure 11. Contours of the offset coherence transformed using (7) against frequency on the abscissa and offset frequency on the ordinate over $780-830 \mu \mathrm{Hz}$ for year-days $268-328$. The time-bandwidth is 5, and nine Slepian tapers were used, yielding 18 degrees of freedom per frequency. The vertical dashed lines denote the center frequencies of the p-modes delineated at the top.

to an increasing frequency density of p-modes. However, the association of high offset coherence with large spectral peaks that occur near the central frequency of p-mode families is ubiquitous.

Further, the magnitude relative to the noise level of a given mode rises with frequency, although this is mitigated to a degree by the fact that the 0.1 quantile overestimates the noise level below $\sim 1,500 \mu \mathrm{Hz}$. There are two $10 \sigma$ and one $8 \sigma$ peaks at $3,700-3,750 \mu \mathrm{Hz}$ in Figure 5; two $8 \sigma$ and one $6 \sigma$ peaks at $2,800-2,870 \mu \mathrm{Hz}$ in Figure S4; one $11 \sigma$ and one $9 \sigma$ peak at $1,745-1,795 \mu \mathrm{Hz}$ in Figure 8 , two $6 \sigma$ peaks at $1,175-1,225 \mu \mathrm{Hz}$ in Figure S6, but only two $5 \sigma$ peaks at $780-830 \mu \mathrm{Hz}$ in Figure 10. This is reflected in the characteristics of the offset coherence that is pervasive over large patches of frequency-offset frequency space at high frequencies (Figures 7 and S5), but subtle at lower frequencies (Figures S7 and 11). Such behavior is consistent with that of the p-modes whose amplitudes rise rapidly at around $2,500 \mu \mathrm{Hz}$ and peak at $2,800-3,400 \mu \mathrm{Hz}$ before falling off to higher frequencies (Christensen-Dalsgaard, 2002; Figure 11).

Another feature of solar p-modes in the pressure field is that only small $m$ splitting appears to be involved even when the degree $l$ is fairly large. This is apparent in Figure 5, where the $\sim 3,704-\mu \mathrm{Hz}$ peak that is possibly associated with $\mathrm{p}_{7,152}$ has a half power bandwidth of $\sim 6 \mu \mathrm{Hz}$ or about three times the resolution bandwidth This corresponds approximately to the frequencies of splitting terms ranging from $-6 \lesssim m \lesssim 6$ despite the allowed range of $-152 \leq m \leq 152$. A similar analysis pertains to the $3,735-\mu \mathrm{Hz}$ peak in the same figure and to the other power spectra plots. The reason for this is not difficult to discern; as the splitting quantum number increases, the active areas on Sun producing modes at Earth become smaller, and also are increasingly concentrated at the solar equator. A shrinking active region that covers a smaller fraction of Sun will be a weaker source of mode energy at Earth as compared to low $m$ modes that cover a substantial fraction of the solar disk.

As described in section 4.1, tidal harmonics appear in the pressure data below $\sim 1,400 \mu \mathrm{Hz}$ and constitute $10-40 \%$ of the noncentral variability at the lower end of the $400-$ to $1,400-\mu \mathrm{Hz}$ bands. Seasonality of the reshaped part of the power spectrum that constitutes tidal harmonics is nearly nonexistent (Table 1), which is not consistent with the mixture $\chi^{2}$ model results in Figure 3, where the noncentral fraction peaks in late summer and exhibits a minimum in winter, although the variability is not marked.

The mixture $\chi^{2}$ model results in Figures 3 and 4 imply about twice as large a noncentral fraction below $1,000-\mu \mathrm{Hz}$ as compared to above $3,000 \mu \mathrm{Hz}$. Taking all of the observations into account, the most consistent explanation for these results is an important contribution from tidal harmonics over $400-1,400 \mu \mathrm{Hz}$ that 
decreases with rising frequency, and predominance of solar modes above $\sim 2,500 \mu \mathrm{Hz}$. However, solar modes are clearly present at intervening frequencies, and in fact the ubiquitous presence of highly significant features with the resolution bandwidth of the estimate occurs across the 400 - to $4,000-\mu \mathrm{Hz}$ bands.

The source locations of tidal-forced IGWs will be relatively small, corresponding to places where the coastal tidal range is largest. However, insight can be obtained by observing how tsunami energy (which overlaps the IGW band of interest in this paper) propagates and reverberates in the ocean basins, homogenizing and becoming isotropic after $\sim 40 \mathrm{hr}$ in the North Pacific Ocean (Van Dorn, 1984). Unlike impulsive tsunamis, tidal forcing is continuous; hence, it may be difficult to clearly identify source locations when the domain is in a persistent state of reverberation. Further work is required on this topic.

It is difficult to further quantify the fraction of solar modes and tidal harmonics in the pressure data because of the pervasive nonstationarity of the former. Nonstationarity results in the transfer of power from frequencies where modal forcing is occurring to other frequencies. There is no constraint that requires that the narrowband characteristics of solar mode peaks be preserved during the power transfer process, and in fact that is what is observed throughout Figures 5, 8, and 10, along with the power spectra in the supporting information. Further, nonstationarity is not limited to the frequency intervals used for analysis and does occur over larger frequency increments. Consequently, it is difficult to separate out mode forcing from nonstationary power transfer, especially at high frequencies where the frequency density of modes becomes large.

It is important to note that a mixture $\chi^{2}$ model can be consistently fit to most frequencies in spectra of the pressure record, implying that the underlying data are approximately Gaussian. Nonstationarity in these data is not strong enough to produce sufficient nonlinearity to lead to non-Gaussianity.

It is well known (e.g., Viall et al., 2009) that the solar wind directly forces magnetospheric disturbances, which then couple to the ionosphere. Both the magnitude of solar modes, as measured through the number of standard deviations from the noise level, and their nonstationarity, as determined from the probability level of the offset coherence, are higher in the geomagnetic field as compared to either the seismic or seafloor pressure fields. This argues for a driving mechanism in which solar modes couple into the magnetosphere/ionosphere, then into the geomagnetic field and finally into other terrestrial observables. Further work is required to verify and quantify this assertion.

In closing, the null hypotheses posed in section 1 that the pressure data are not influenced by solar normal modes (tidal harmonics) in the IGW band must be rejected. Solar modes explain as much as approximately one third of the data variance over $400-4,000 \mu \mathrm{Hz}$. In addition, tidal harmonics are present at up to $\sim 1,400 \mu \mathrm{Hz}$ and explain up to $40 \%$ of the variance over that band. Finally, none of the analyses presented in this paper refute the existence of additional sources for IGW variability in the deep ocean, such as that proposed by Webb et al. (1991). Rather, the results show that IGWs in the deep ocean are complex phenomena with multiple sources.

\section{Conclusions}

It is well established in statistics that the power spectral density of a stationary process is uncorrelated at distinct frequencies, while that of a nonstationary process is correlated across frequency. Further, nonstationarity implies that forcing at a given frequency will result in the transfer of power to other frequencies, and such a behavior is inherently nonlinear. In this paper, these characteristics of stationary and nonstationary processes are applied to understand the fluctuations of the deep ocean pressure field over the IGW band.

IGWs have arguably received the least study of any class of waves in the deep ocean. This paper contains the analysis of a 389-day-long deep ocean pressure record collected north-northwest of Kauai in the North Pacific Ocean during the Hawaii Ocean Mixing Experiment for narrowband components and nonstationarity over $400-4,000 \mu \mathrm{Hz}$ using a combination of fitting a mixture noncentral/central $\chi^{2}$ model to spectral estimates, high-resolution multitaper spectral estimation, and computation of the offset coherence between distinct frequencies for a given data segment. In the frequency band $400-1,000 \mu \mathrm{Hz}$, a mixture noncentral/central $\chi^{2}$ model is fit to the spectral estimates, showing a noncentral fraction of $0.67 \pm 0.07$ that decreases with increasing frequency, vanishing beyond $\sim 1,400 \mu \mathrm{Hz}$. Evidence is presented for the presence 
of tidal harmonics in the data over the $400-$ to $1,400-\mu \mathrm{Hz}$ bands, possibly due to a coastal nonlinear energy cascade originating in the Gulf of Alaska. The mixture $\chi^{2}$ model can also be fit to the spectral estimates beyond $\sim 2,000 \mu \mathrm{Hz}$, with the noncentral fraction rising with frequency and comprising about one third of the spectral estimates over $3000-4000 \mu \mathrm{Hz}$.

Over the 3,700 to $3,750-\mu \mathrm{Hz}$ bands, there are two peaks at the $2 \mu \mathrm{Hz}$ resolution bandwidth of the estimate that exceed 11 standard deviations from the noise level, and an additional peak that exceeds 9 standard deviations; the estimated $Q$ for these peaks is over 1,000. The probability of independently observing three such peaks is $3.9 \times 10^{-10}$, which is effectively impossible unless there is a nonrandom component present. This strongly argues for the presence of quasi-harmonic forcing at one or more frequencies in the analysis band. The corresponding offset coherence is quite striking in that symmetric trapezoidal blocks with $p=0.9999$ appear about zero offset frequency at each of the peaks in the power spectrum. These have an internal structure that suggests that each of the peaks comprises numerous narrowband components that are themselves coherent. Trapezoidal blocks also occur at offset frequencies consistent with coherence between the peaks. The picture that emerges is one of nonstationary behavior that is manifest both as high offset coherence within spectral peaks and between them.

The power spectrum over the frequency band $1,745-1,795 \mu \mathrm{Hz}$ exhibits a single peak that is $11 \sigma$ above the noise level, followed by a $9 \sigma$ peak, a $7 \sigma$ peak, and two $4 \sigma$ ones. The probability of independently observing the two largest peaks in a central $\chi_{18}^{2}$ population is $4.6 \times 10^{-8}$, and for the three largest peaks it is $3.2 \times 10^{-10}$. Either of these scenarios is extremely unlikely, and so the presence of a nonrandom component in the pressure data is assured. The peaks have a full bandwidth at half power of $\sim 2 \mu \mathrm{Hz}$, implying a $Q$ of at least 890 . Further, most of the peaks occur near the center $(m=0)$ frequency of solar p-modes. The corresponding offset coherence exhibits a very large trapezoidal coherent patch in the middle of the plot extending from $\sim 1,758$ to $1,785 \mu \mathrm{Hz}$ along zero offset frequency, indicating pervasive coherence over the middle two thirds of the power spectrum.

The power spectrum at $780-830 \mu \mathrm{Hz}$ exhibits five narrow peaks over the $50-\mu \mathrm{Hz}$ analysis band that exceeds the noise level by at least $4 \sigma$, and two peaks that exceed it by nearly $6 \sigma$, and all have a bandwidth comparable to the resolution value. The probability of five peaks occurring independently above the $4 \sigma$ level is $2.2 \times 10^{-5}$, which is very unlikely unless there is a nonrandom component present. Two of the spectral peaks occur at harmonics of tidal species and do not have a significant offset coherence. Analysis of the offset coherence and spectral density unambiguously establishes the existence of forcing at the frequencies of the $\mathrm{p}_{4,2}$ solar mode, along with the transfer of power to other frequencies due to nonstationarity.

Solar p-modes are readily detectable throughout the 400 - to $4,000-\mu \mathrm{Hz}$ analysis band, as seen through the appearance of features with a $Q$ whose lower bound is determined by the resolution bandwidth of the estimate, yet with a value much higher than that of known terrestrial or oceanic phenomena, and in close association with the known frequencies of solar p-modes. Tidal harmonics appear in the pressure data below $\sim 1,400 \mu \mathrm{Hz}$ and constitute up to $40 \%$ of the noncentral variability at the lower end of the 400 to $1,400 \mu \mathrm{Hz}$ bands. It is difficult to further quantify the fraction of solar modes and tidal harmonics in the pressure data because of the pervasive nonstationarity of the former. Nonstationarity results in the transfer of power from frequencies where modal forcing is occurring to other frequencies. There is no constraint that requires that the narrowband characteristics of solar mode peaks be preserved during the power transfer process. Further, nonstationarity is not limited to the frequency intervals used for analysis and does occur over larger frequency increments. Consequently, it is difficult to separate out mode

Acknowledgments

This work was supported at WHOI by an Independent Research and Development award and the Walter A. and Hope Noyes Smith Chair for Excellence in Oceanography. At the University of Hawaii, Martin Guiles provided a number of consequential data analyses, and work was supported by NSF-OCE1460022. D. J. T. acknowledges support from Queen's University and NSERC. The data used in this study are available from the supporting information. forcing from nonstationary power transfer, especially at high frequencies where the frequency density of modes becomes large.

\section{References}

Aki, K. (1957). Space and time spectra of stationary stochastic waves, with special reference to microtremors. Bulletin of the Earthquake Research Institute Tokyo, 35, 415-457.

Aucan, J., \& Ardhuin, F. (2013). Infragravity waves in the deep ocean: An upward revision. Geophysical Research Letters, 40, 3435-3439. https://doi.org/10.1002/grl.50321

Chave, A. D. (2017). Computational statistics in the Earth sciences. Cambridge: Cambridge U. Press.

Christensen-Dalsgaard, J. (2002). Helioseismology. Reviews of Modern Physics, 74, 1073-1129.

Cramér, H. (1940). On the theory of stationary random processes. Annals of Mathematics, 41, 215-230. 
Elgar, S., Herbers, T. H. C., Okihiro, M., Oltman-Shay, J., \& Guza, R. T. (1992). Observations of infragravity waves. Journal of Geophysical Research, 87, 457-468

Filloux, J. H. (1971). Deep sea tide observations from the northeastern Pacific. Deep Sea Research, 18, $275-284$.

Filloux, J. H. (1980). Pressure fluctuations on the open ocean floor over a broad frequency range: New program and early results. Journal of Physical Oceanography, 10, 1959-1971.

Filloux, J. H. (1983). Pressure fluctuations on the open-ocean floor off the Gulf of California: Tides, earthquakes, tsunamis. Journal of Physical Oceanography, 13, 783-796.

Filloux, J. H., Luther, D. S., \& Chave, A. D. (1991). Long-term seafloor measurements of water pressure: Normal modes and infragravity waves, IAPSO Proceedings No. 18 for IUGG XXth General Assembly, Vienna, Austria, 11-24 August, 1991, p. 299.

Godin, O. A., Zabotin, N. A., Sheehan, A. F., Yang, Z., \& Collins, J. A. (2013). Power spectra of infragravity waves in a deep ocean. Geophysical Research Letters, 40, 2159-2165. https://doi.org/10.1002/grl.50418

Hanssen, A., Larsen, Y., \& Scharf, L. L. (2004). Complex time-frequency and dual-frequency spectra of harmonizable processes. Proc. 12th Eur. Signal Proc. Conf., 1577-1580.

Leffler, K. E., \& Jay, D. A. (2009). Enhancing tidal harmonic analysis: Robust (hybrid L1/L2) solutions. Continental Shelf Research, 29(1), 78-88. https://doi.org/10.1016/j.csr.2008.04.011

Loève, M. (1945). Sur les fonctions aléatoires stationnaires de second ordre. La Revue Scientifique, 83, $297-303$.

Loève, M. (1946). Fonctions aléatoires du second ordre. La Revue Scientifique, 84, 195-206.

Luther, D. S., Chave, A. D., \& Webb, S. C. (2014). Pelagic infragravity waves forced by tidal non-linear Interactions. AGU Ocean Sciences Meeting, February 23-28, 2014, Honolulu, HI. (Abstract ID: 15452).

Mallows, C. L. (1967). Linear processes are nearly Gaussian. Journal Applied Probability, 4(02), 313-329. https://doi.org/10.2307/ 3212026

Masters, T. G., \& Widmer, R. (1995). Free oscillations: Frequencies and attenuations. In T. J. Ahrens (Ed.), Global Earth physics (pp. 104-125). Am. Geophys. Union: Wash. DC.

Munk, W., Snodgrass, F., \& Gilbert, J. F. (1964). Long waves on the continental shelf: An experiment to separate trapped and leaky modes. Journal of Fluid Mechanics, 20(04), 529-554. https://doi.org/10.1017/S0022112064001392

Nawa, K., Suda, N., Fukao, Y., Sato, T., Aoyama, Y., \& Shibuya, K. (1998). Incessant excitation of the Earth's free oscillations. Earth, Planets and Space, 50(1), 3-8. https://doi.org/10.1186/BF03352080

Nishida, K., Kobayashi, N., \& Fukao, Y. (2000). Resonant oscillations between the solid earth and atmosphere. Science, 287(5461), 2244-2246. https://doi.org/10.1126/science.287.5461.2244

Pinkel, R., Munk, W., Worcester, P., Comuelle, B. D., Rudnick, D., Sherman, J., et al. (2000). Ocean mixing studied near Hawaiian Ridge. EOS Trans AGU, 81(46), 545-553. https://doi.org/10.1029/EO081i046p00545-02

Provost, J., Barthomieu, G., \& Morel, P. (2000). Low-frequency p- and g-mode solar oscillations. Astronomy \& Astrophysics, 353, 775-785.

Ray, R. D. (2007). Propagation of the overtide M4 through the deep Atlantic Ocean. Geophysical Research Letters, 34, L21602. https://doi. org/10.1029/2007GL031618

Rhie, J., \& Romanowicz, B. (2004). Excitation of Earth's continuous free oscillations by atmosphere-ocean-seafloor coupling. Nature, 431(7008), 552-556. https://doi.org/10.1038/nature02942

Rhodes, E. J. Jr., Kosovichev, A. G., Schou, J., Scherrer, P. H., \& Reiter, J. (1997). Measurements of frequencies of solar oscillations from the MDI medium-l program. Solar Physics, 175(2), 287-310. https://doi.org/10.1023/A:1004963425123

Schreier, P. J., \& Scharf, L. L. (2010). Statistical signal processing of complex-valued data. Cambridge: Cambridge U. Press.

Smith, M. F., \& Masters, G. (1989). Aspherical structure constraints from free oscillation frequency and attenuation measurements. Journal of Geophysical Research, 94(B2), 1953-1976. https://doi.org/10.1029/JB094iB02p01953

Thomson, D. J. (2001). Multitaper analysis of nonstationary and nonlinear time series data. In W. Fitzgerald, R. Smith, A. Walden, \& P. Young (Eds.), Nonlinear and Nonstationary Signal Processing (pp. 317-394). Cambridge: Cambridge University Press.

Thomson, D. J., \& Chave, A. D. (1991). Jackknife error estimates for spectra, coherences, and transfer functions. In S. Haykin (Ed.), Advances in spectral analysis and array processing, (Vol. 1, pp. 58-113). Englewood Cliffs: Prentice-Hall.

Thomson, D. J., Lanzerotti, L. J., \& Maclennan, C. G. (2001). The interplanetary magnetic field: Statistical properties and discrete modes. Journal of Geophysical Research, 106(A8), 15,941-15,962. https://doi.org/10.1029/2000JA000113

Thomson, D. J., Lanzerotti, L. J., Vernon, F. L. III, Lessard, M. R., \& Smith, L. T. P. (2007). Solar modal structure of the engineering environment. Proceedings of the IEEE, 95(5), 1085-1132. https://doi.org/10.1109/JPROC.2007.894712

Thomson, D. J., Maclennan, C. G., \& Lanzerotti, L. J. (1995). Propagation of solar oscillations through the interplanetary medium. Nature, 376(6536), 139-144. https://doi.org/10.1038/376139a0

Thomson, D. J., \& Vernon, F. L. III (2015). Unexpected, high-Q, low-frequency peaks in seismic spectra. Geophysical Journal International, 202(3), 1690-1710. https://doi.org/10.1093/gji/ggv175

Thomson, D. J., \& Vernon, F. L. III (2016). Some comments on the analysis of "big" scientific time series. Proceedings of the IEEE, 104(11), 2220-2249. https://doi.org/10.1109/JPROC.2016.2598218

Uchiyama, Y., \& McWilliams, J. C. (2008). Infragravity waves in the deep ocean: Generation, propagation and seismic hum excitation. Journal of Geophysical Research, 113, C07029. https://doi.org/10.1029/2007JC004562

Van Dorn, W. G. (1984). Some tsunami characteristics deducible from tide records. Journal of Physical Oceanography, 14(2), 353-363. https://doi.org/10.1175/1520-0485(1984)014<0353:STCDFT>2.0.CO;2

Viall, N. M., Kepko, L., \& Spence, H. E. (2009). Relative occurrence rates and connection of discrete frequency in the solar wind density and dayside magnetosphere. Journal of Geophysical Research, 114, A01201. https://doi.org/10.1029/2008JA013334

Webb, S. C. (1986). Coherent pressure fluctuations observed at two sites on the deep sea floor. Geophysical Research Letters, 13(2), 141-144. https://doi.org/10.1029/GL013i002p00141

Webb, S. C. (2007). The Earth's 'hum' is driven by ocean waves over the continental shelves. Nature, 445(7129), 754-756. https://doi.org/ 10.1038 /nature05536

Webb, S. C. (2008). The Earth's hum: The excitation of Earth normal modes by ocean waves. Geophysical Journal International, 174(2), 542-566. https://doi.org/10.1111/j.1365-246X.2008.03801.x

Webb, S. C., Zhang, X., \& Crawford, W. (1991). Infragravity waves in the deep ocean. Journal of Geophysical Research, 96(C2), 2723-2736. https://doi.org/10.1029/90JC02212

Yu, X., Crossley, D., \& Hermann, R. B. (2008). Amplitude and Q of ${ }_{0} \mathrm{~S}_{0}$ from the Sumatra earthquake as recorded on superconducting gravimeters and seismometers. Seismological Research Letters, 79, 797-805. 


\section{References From the Supporting Information}

Aki, K. (1965). A note on the use of microseisms in determining the shallow structures of the earth's crust. Geophysics, $30,665-666$. Chaplin, W. J., Appourchaux, T., Elsworth, Y., Isaak, G. R., Miller, B. A., \& New, R. (2000). Source of excitation of low-l solar p modes: Characteristics and solar-cycle variations. Monthly Notices of the Royal Astronomical Society, 314, 75-86.

Dahlen, F. A., \& Tromp, J. (1998). Theoretical global seismology. Princeton: Princeton Univ. Press.

Moretti, P. F., Cacciani, A., Hanslmeier, A., Messerotti, M., Oliviero, W., Ortuba, W., et al. (2001). The source of solar oscillations: Convective or magnetic. Astronomy and Astrophysics, 372(3), 1038-1047. https://doi.org/10.1051/0004-6361:20010588

Percival, D. B., \& Walden, A. T. (1993). Spectral analysis for physical applications. Cambridge: Cambridge U. Press.

Scherrer, P. H., Bogart, R. S., Bush, R. I., Hoeksema, J. T., Kosovichev, A. G., Schou, J., et al., \& the MDI Engineering Team (1995). In B. Fleck, V. Domingo, \& A. Poland (Eds.), The Soho Mission. Dordrecht: Springer.

Slepian, D. (1978). Prolate spheroidal wave functions, Fourier analysis and uncertainty-V. The discrete case. Bell System Technical Journal, 57(5), 1371-1430. https://doi.org/10.1002/j.1538-7305.1978.tb02104.x

Stix, M. (2004). The Sun: An Introduction, (2nd ed.). Berlin: Springer-Verlag.

Thomson, D. J. (1982). Spectrum estimation and harmonic analysis. Proceedings of the. IEEE, 70(9), 1055-1096. https://doi.org/10.1109/ PROC.1982.12433

Woodard, M. F., \& Noyes, R. W. (1985). Change in solar oscillation eigenfrequencies with the solar cycle. Nature, 318(6045), 449-450. https://doi.org/10.1038/318449a0 